\title{
PROGRESS
}

\section{Translation inhibition and metabolic stress pathways in the host response to bacterial pathogens}

\section{Bruno Lemaitre and Stephen E. Girardin}

Abstract | Activation of most major innate immune signalling cascades relies on the detection of microorganisms or their associated danger signals by host pattern recognition molecules. A flurry of recent studies has now uncovered a role for host translation inhibition in innate immune surveillance and the detection of bacterial pathogens. Here, we present the main findings from these studies and discuss whether translation inhibition is an alarm signal that directly drives innate immune responses to bacterial pathogens, or rather one component of a more general metabolic stress response to infection.

Innate immunity protects multicellular organisms against infection by triggering host protective and defence responses against pathogens. Numerous families of host extracellular and intracellular sensors of microorganisms have been identified, and these include Toll-like receptors (TLRs) and NOD-like receptors (NLRs) in vertebrates, and the peptidoglycan recognition proteins (PGRPs) that trigger the Toll and immune deficiency (Imd) pathways in Drosophila melanogaster ${ }^{1-3}$. These host pattern recognition receptors (PRRs) detect conserved microbial molecular signatures called microorganism-associated molecular patterns (MAMPs), such as bacterial lipopolysaccharide (LPS), peptidoglycan, flagellin and CpG DNA, as well as viral double- or single-stranded RNA (see REF. 4 for a comprehensive recent review on this topic). More recently, it has become evident that pattern recognition of microbial determinants is not sufficient to explain the activation of the full spectrum of innate immune defence programmes. Indeed, metazoans also harbour receptors that actually detect cellular damage or perturbations which can be triggered by pathogens, rather than the microbial molecules per se $\mathrm{e}^{5,6}$. Such signals, known as danger signals or danger-associated molecular patterns (DAMPs), include potassium efflux, reactive oxygen species
(ROS), lysosomal damage, molecular crystals, and some xenocompounds and molecules released by dying cells, such as uric acid or mitochondrial DNA. The idea that DAMPs can serve as an initiating signal for innate immunity is conceptually related to a central theme in plant immunity known as effector-triggered immunity (ETI), a theme which suggests that the infected plant host can detect host molecule alterations that are induced by bacterial effectors?

The detection of perturbations in infected cells might be functionally as important for the detection and control of pathogens as detection of the microorganism itself. A series of studies published over the past 2 years is now starting to shape an unexpected new dimension to this emerging concept by showing that host gene translation (or the pathways controlling this process) is a crucial cellular process with an activity that seems to be tightly associated with host innate immune defences ${ }^{8-14}$.

Host translation inhibition is a central element of defence against viral infection ${ }^{15}$. Although this defence response is logical, given that viruses need to hijack the host translation machinery to produce polypeptides, it seems less obvious in the case of bacterial pathogens, which have their own translation machineries. The observation that bacterial infection can lead to translation inhibition is therefore probably an indication that this process is aimed at providing cellular adaptation to the infection, rather than being directed against the pathogen itself.

In this Progress article, we summarize the main findings from these recent studies using different model systems. We also question whether translation inhibition is genuinely a signal associated with innate immune control of bacterial pathogens or whether this process is only one aspect of a more general metabolic stress response to infection.

\section{L. pneumophila in mammalian cells}

Legionella pneumophila is a Gram-negative bacterial pathogen that is a parasite of amoebae and accidentally infects human hosts as a result of inhalation of water aerosols, causing Legionnaires' disease and Pontiac fever. L. pneumophila invades human macrophages through the Dot/Icm (defect in organelle trafficking/intracellular multiplication) type IV secretion system, which enables the translocation of numerous bacterial effectors that hijack key host cellular function $s^{16}$. Using a comparative microarray analysis, Fontana et al. identified a number of mouse macrophage genes that were upregulated on macrophage infection with wild-type L. pneumophila but not on infection with a $\triangle \operatorname{dot} A$ mutant lacking a functional Dot/Icm secretion system ${ }^{10}$. As both wild-type and $\Delta \operatorname{dot} A$ L. pneumophila are expected to express similar MAMPs, the authors speculated that the host genes responding differentially to these two bacteria are modulated by a specific stress that is dependent on the activity of particular bacterial effectors, and that the upregulation of these genes thus corresponds to a form of ETI. As L. pneumophila encodes five effectors that are known to inhibit the host mRNA translation machinery ${ }^{17}$, it was postulated that alteration of host translation could be such a stress. In support of this proposal, an L. pneumophila strain lacking all five effectors behaved similarly to the $\Delta \operatorname{dot} A$ strain $^{10}$.

Pharmacological inhibitors of host translation initiation or elongation induced the transcription of a selection of genes that the authors had identified as components 


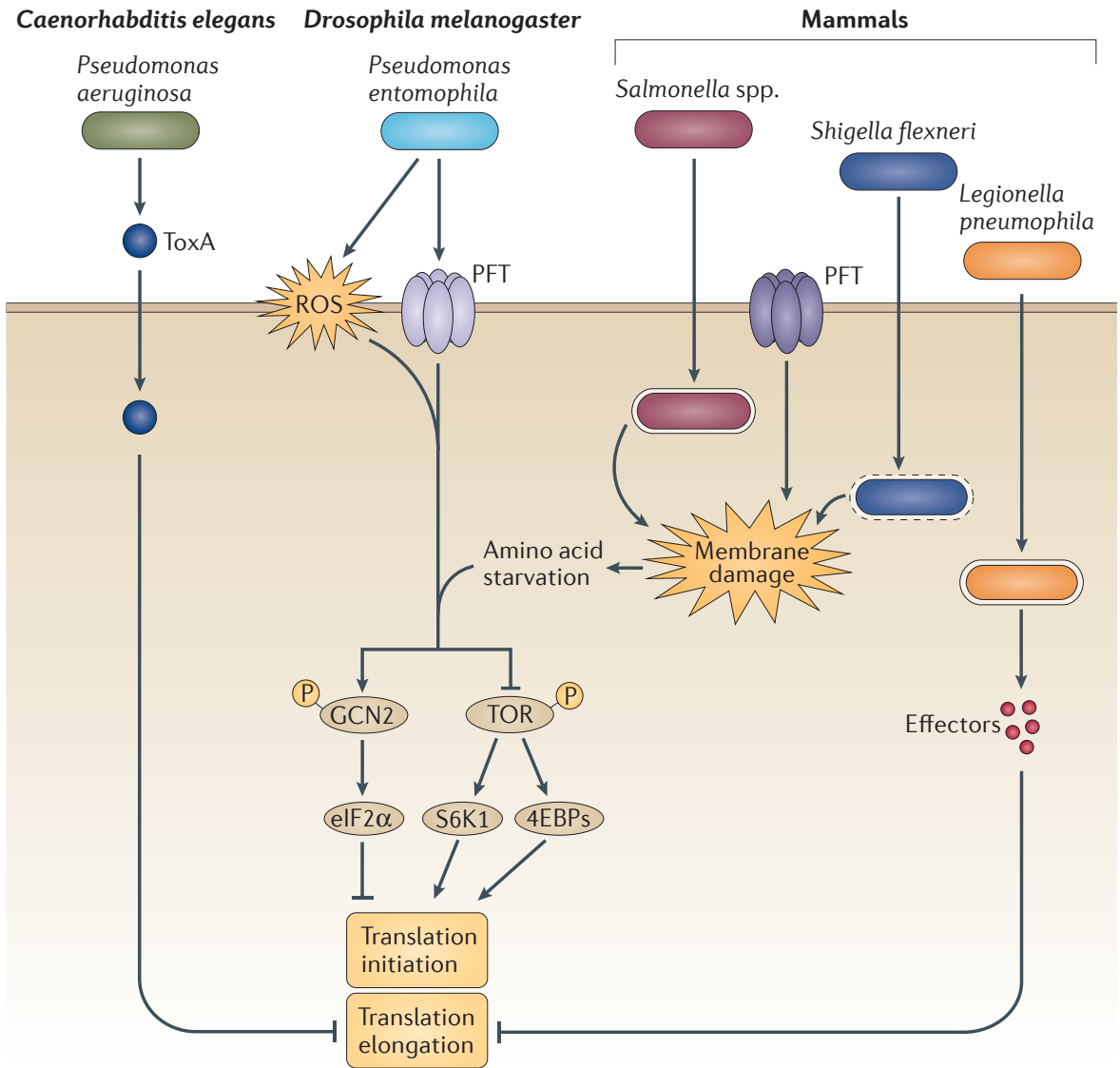

Figure 1 | Translation inhibition by bacterial pathogens. Schematic representation of the various mechanisms through which bacterial infection can result in inhibition of the translation machinery, either directly (through bacterial toxin- or effector-mediated inhibition of translation elongation) or indirectly (through activation of the GCN2 pathway, which inhibits translation initiation, or inactivation of the target of rapamycin (TOR) pathway, which would otherwise promote translation initiation). Poreforming toxins (PFTs) are produced by Pseudomonas entomophila and other species, including Staphylococcus aureus (a-toxin), Vibrio cholerae (cytolysin), Streptococcus pneumoniae (streptolysin O) and Escherichia coli (haemolysin). 4EBPs, elF4E-binding proteins; elF2 $\alpha$, eukaryotic translation initiation factor 2 subunit- $\alpha$; ROS, reactive oxygen species; S6K1, ribosomal protein S6 kinase $\beta 1$; ToxA, exotoxin $\mathrm{A}$.

of the ETI response when uninfected host cells were incubated in the presence of the MAMP PamCSK4 (a synthetic lipopeptide ligand for TLR2). The authors obtained similar results using two bacterial ADPribosyltransferases (diphtheria toxin from Corynebacterium diphtheriae and exotoxin A (ToxA) from Pseudomonas aeruginosa) that target elongation factor 2 (EF2), a highly conserved component of the host translation elongation machinery. Thus, the host transcriptomic profile observed on L. pneumophila infection results from the integration of two signals, one emanating from MAMP-dependent recognition and the other from inhibition of translation as a consequence of the ETI response (FIG. 1). The authors also demonstrated that NF- $k B$

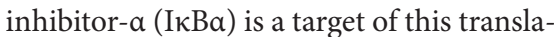
tion inhibition, thus potentially accounting for nuclear factor- $\mathrm{kB}$ (NF-kB)-associated differences in the response of macrophages to wild-type or $\Delta \operatorname{dot} A$ L. pneumophila.

\section{C. elegans model of infection}

The nematode Caenorhabditis elegans lacks specific immune cells, implying that all C. elegans cells, and in particular those in direct contact with the environment (such as epithelial cells), must be equipped with efficient means to control microbial pathogens. C. elegans also lacks many of the immune signalling pathways found in other metazoans (such as the NF- $\mathrm{kB}$ and Janus-activated kinase-signal transducer and activator of transcription (JAK-STAT) pathways) and lacks PRR families that have been identified in other animal species, such as TLRs and NLRs. Therefore, it remains a key aim to understand how C. elegans mounts a defence response against infection with harmful microorganisms. Moreover, defining how this model organism can differentiate between harmless and pathogenic bacteria is of particular importance, given that this animal feeds on bacteria that are frequently found in rotting fruits, where it resides.

Previous work had shown that the expression of $C$. elegans infection response gene 1 (irg-1) is triggered in response to pathogenic $P$. aeruginosa (a bacterium that causes lethal infection in C. elegans), but not in response to a non-pathogenic mutant strain lacking many virulence factors ${ }^{18}$. It had also been found that the pathway leading to irg- 1 induction is dependent on a basic leucine zipper (bZIP) transcription factor named ZIP-2 and that activation of the ZIP-2-IRG-1 signalling axis is crucial for host defence ${ }^{18}$. In a recent study by Dunbar et al. building on this previous work, an RNAi screen revealed that inhibition of diverse basic cellular functions, such as translation, metabolic enzymatic processes or fatty acid metabolism, induces the ZIP-2IRG-1 pathway, suggesting that $P$. aeruginosamediated induction of this signalling axis is indirect, occurring as a result of the inhibition of core cellular functions?.

P. aeruginosa ToxA is an ADPribosyltransferase that shuts off host translation elongation by inhibiting host EF2 (REF. 17). Dunbar et al. observed that infection of C. elegans with wild-type P. aeruginosa, but not a toxA-deficient strain, efficiently suppressed the expression of conditionally induced GFP in vivo ${ }^{9}$. However, toxA-deficient $P$. aeruginosa still triggered irg-1 expression in infected C. elegans. This important finding suggests that host translation inhibition by $P$. aeruginosa is not the only mechanism contributing to irg- 1 induction.

Finally, Dunbar et al. studied the paradoxical induction of zip-2 mRNA translation under conditions of translation arrest. The authors observed the presence of upstream ORFs (uORFs) that compete with the main ORF in the $5^{\prime}$ UTR of zip-2 mRNA, and then demonstrated the in vivo importance of these uORFs in the regulation of $z i p-2$ expression in P. aeruginosa-infected animals. Interestingly, this translation regulation mechanism bears some similarities to the mechanism that has been extensively studied for mammalian AMP-dependent transcription factor $4(\mathrm{ATF} 4)^{19}$, another bZIP protein that is induced by translation stress.

In a related study, McEwan et al. analysed the response of $C$. elegans to $P$. aeruginosa ToxA ${ }^{12}$. Using comparative microarrays, the authors identified 144 genes that were 
upregulated more than twofold in response to ToxA, $\sim 50 \%$ of which were also induced by $P$. aeruginosa infection. The transcriptional signature associated with exposure to ToxA did not represent a general response to all types of infection, because it had only partial overlap with the response to Candida albicans or Staphylococcus aureus. The ToxAinduced genes were shown to be dependent on some of the signalling pathways that are involved in the response to $P$. aeruginosa, such as the ZIP-2-IRG-1 pathway and the p38 mitogen-activated protein kinase 1 (PMK-1) pathway. Moreover, survival assays demonstrated that these two pathways have key roles in the response of C. elegans to the toxicity of ToxA. Importantly, McEwan et al. found that a catalytically inactive ToxA failed to trigger an irg-1::GFP reporter in C. elegans, implying that it is the translation arrest property of ToxA, rather than the capacity of the host to detect this molecule per se, which is crucial for the induction of irg-1. In support of this, the transcriptional signature of $C$. elegans after exposure to the translation inhibitor hygromycin had similarities to the transcriptional signature triggered by ToxA.

In a separate study, Melo et al. did not focus exclusively on the response to a specific bacterial pathogen or toxin ${ }^{13}$, but instead used an RNAi library of doublestranded RNAs that were ectopically expressed in Escherichia coli (the bacterium used as the food substratum for C. elegans) in order to inactivate 4,062 essential and metabolic genes in C. elegans. The authors screened for RNAi targets that triggered aversion of C. elegans to the bacterial food, as a way of identifying putative danger signals - in the form of alterations to the core C. elegans cellular machineries - which alert the animal that the microbial food source could be pathogenic and should be avoided. They observed that this microbialaversion phenotype was triggered following inhibition of several cellular processes, including translation and mitochondrial or proteasomal activity. In agreement with these findings, feeding C. elegans with lawns of non-pathogenic E. coli supplemented with poisons that target these essential cellular processes also triggered the aversion behaviour. Finally, RNAi-mediated inhibition of key host cellular processes also activated GFP reporters under the transcriptional control of clec-60, nlp-29 and irg-1, three genes known to be induced by pathogens.

Together, these three studies suggest that, in the possible absence of a bona fide PRR system, C. elegans has evolved the capacity to mount a defence against ingested pathogenic bacteria in the gut by detecting the action of their virulence factors on vital cellular processes, including translation (FIG. 2).

\section{D. melanogaster model of infection}

In D. melanogaster, efficient and rapid recovery from bacterial infection in the gut requires not only bacterial clearance by the immune system, but also repair of the intestine through renewal of the epithelium damaged by infection ${ }^{20,21}$. In a recent investigation, Chakrabarti et al. analysed how the entomopathogenic bacterium Pseudomonas entomophila disrupts gut homeostasis, revealing a central role for translation inhibition in P. entomophila pathogenesis ${ }^{8}$.

\section{P. entomophila ingestion by D. melano-} gaster was found to stimulate the expression of antimicrobial peptide genes through the Imd pathway. However, this immune response was not productive owing to a general inhibition of translation that affected all newly synthesized transcripts. This not only stopped the immune response, but also inhibited the repair programme by blocking signals that stimulate intestinal stem cells. As a consequence, $D$. melanogaster succumbed to $P$. entomophila infection because it was unable to repair gut damage. The authors showed that in this in vivo model of infection the reduction of translation is a consequence of cellular damage to the intestine, caused by both host-derived reactive oxygen species and the direct action of a pore-forming toxin produced by the pathogen (FIG. 1).

What are the pathways that link this cellular damage to the reduction of translation? In eukaryotes, cap-dependent protein synthesis is typically controlled by many regulatory proteins that are themselves under the control of stress-responsive pathways. Using a genetic approach, Chakrabarti et al. showed that inhibition of translation is induced by two signalling pathways: the phosphorylation of eukaryotic translation initiation factor 2 subunit- $\alpha$ (eIF2 $\alpha$ ) by the stress kinase $\mathrm{Gcn} 2$, and inhibition of the target of rapamycin (Tor) pathway by AMPactivated protein kinase (AMPK; also known as SNF1A). Both kinases sense metabolic deprivation (amino acids for Gcn 2 and energy for AMPK), suggesting that cellular damage induced by $P$. entomophila in turn induces a state of 'starvation'. Inhibition of translation is usually an adaptive cellular response to adjust the metabolism to the energy status of the cells. The observation that Gcn2-deficient D. melanogaster survived $P$. entomophila infection better than wild-type $D$. melanogaster indicates that a Caenorhabditis elegans

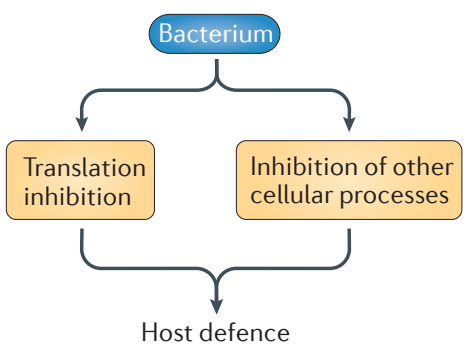

b Drosophila melanogaster and mammals

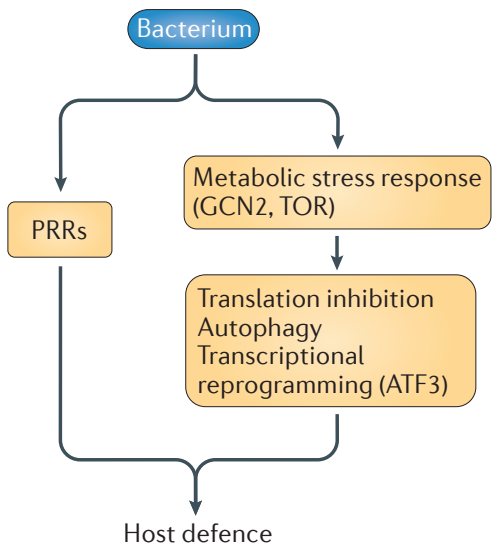

Figure 2 | Translation inhibition in the context of host defence against bacterial pathogens. a | The current model suggests that inhibition of translation, among other core cellular processes, contributes to host defence in Caenorhabditis elegans. It remains unclear whether unidentified pattern recognition receptors (PRRs) also contribute to host defence in C. elegans. b| In Drosophila melanogaster and mammals, host defence requires signals from PRRs and metabolic stress (through the GCN2 and target of rapamycin (TOR) pathways). In these organisms, translation inhibition thus represents one arm of the metabolic stress response to bacterial pathogens.

pathogenesis is linked to overactivation of the stress pathways that usually help cells to endure the consequences of an infection.

Inhibition of translation also had an influence on the innate immune response observed on P. entomophila infection; this response was characterized by the systemic production of antimicrobial peptides by the fat body. The same pattern of immune response was observed when D. melanogaster was fed with a non-lethal pathogen in combination with an inhibitor of translation. Chakrabarti et al. hypothesize that, in line with the results obtained by Fontana et al. in mammalian cells ${ }^{10}$, translation inhibition in D. melanogaster blocks the synthesis of negative regulators of the Imd pathway (PGRPLB and Pirk), resulting in a systemic immune response to oral infection. Finally, 
the authors speculate that the rate of protein synthesis acts as a direct sensor of pathogenencoded activities and modulates the immune response through short-lived negative regulators. Such a sensing system would reflect the global level of cellular stress and therefore act in response to any type of infectious damage, rather than recognizing a specific pathogen effector.

\section{Invasive bacteria in mammalian cells}

Shigella flexneri and Salmonella enterica subsp. enterica serovar Typhimurium use specific effectors to invade mammalian cells. However, in contrast with the above studies, these bacterial pathogens do not encode $\mathrm{AB}$ or pore-forming toxins that would directly or indirectly, respectively, target the host translation machinery. Tattoli et al. showed that $S$. flexneri infection of human epithelial cells triggers an acute and sustained state of amino acid starvation characterized by inhibition of the metabolic checkpoint kinase mammalian TOR (mTOR), as well as induction of the amino acid stress pathway dependent on GCN2, eIF2 $\alpha$ and the bZIP protein ATF3 (REF. 14) (FIG. 1). Using microarrays, the authors demonstrated that, in addition to the prototypical inflammatory response driven by NF- $\kappa \mathrm{B}$ signalling, $S$. flexneri infection triggers a transcriptional stress signature associated with the induction of ATF3 expression. In the case of $S$. Typhimurium, this stress response was only transient and was associated with the hijacking of mTOR complex 1 to recruit it to the surface of the Salmonella-containing vacuole, suggesting that the pathogen has evolved a means to subvert metabolic stress defence pathways.

The authors next demonstrated that host membrane damage is sufficient to trigger amino acid starvation. This is in agreement with studies that report the induction of similar pathways by bacterial pore-forming toxins from various structural families ${ }^{22,23}$, and is also consistent with the role of the $P$. entomophila pore-forming toxin in the induction of the Tor and Gcn2 pathways in infected $D$. melanogaster (see above). In a follow-up study, it was found that in the case of $S$. Typhimurium, the membrane damage is caused by Salmonella pathogenicity island 1 and is thus most probably driven by the insertion of the type III secretion system into the membrane of the Salmonella-containing vacuole $^{24}$.

Finally, Tattoli et al. determined that one of the functional consequences of the induction of host amino acid starvation is to promote autophagy directed against the intracellular bacteria, thus controlling the infection $^{14}$. Interestingly, the bacterium-induced membrane damage seems to have a dual role in the coordination and execution of antibacterial autophagy, as it simultaneously triggered metabolic stress (amino acid starvation) and allowed the recruitment of autophagytargeting factors, such as NDP52 (also known as CALCOCO2).

\section{Concluding remarks and perspectives}

It is puzzling why host translation inhibition during bacterial infection was not studied more extensively in the past, when most of the main signal transduction pathways associated with host defence against bacterial infection were identified in both mammals and invertebrates. However, most readouts used in cellular microbiology rely on analysis of the transcriptional output of infection, potentially ignoring the numerous post-transcriptional events. Also, the recent articles described above clearly link bacterial infection with translation inhibition only in the case of pathogens expressing specific $\mathrm{AB}$ or pore-forming toxins. Moreover, it is also important to consider that these studies show only a partial overlap between the effects of bacterial infection and the effects of general translation inhibitors, such as cycloheximide. This suggests that, in the physiological context of infection, translation inhibition is partial or affects only a subset of genes.

The results obtained in the C. elegans model seem to argue for a specific role of translation inhibition pathways in host defence against bacterial pathogens. However, this interpretation must be considered with caution. The studies by Dunbar et al. ${ }^{9}$ and McEwan et al. ${ }^{12}$ used a bacterial pathogen ( $P$. aeruginosa) that expresses a toxin which inhibits translation, and in order to generalize these findings as a generic innate immune mechanism, it will be important to analyse translation inhibition using other natural C. elegans pathogens that do not express such toxins. In addition, it will be important to determine whether translation inhibition can directly trigger the expression of conserved sets of antimicrobial genes in C. elegans. In support of this scenario is the observation by Melo et al. that pathogen-responsive genes (including the antimicrobial peptide NLP-29) are induced by inactivation of core cellular functions, including translation ${ }^{13}$.

When considering the potential importance of translation inhibition in innate immune defence, it is crucial to distinguish between situations in which translation is inhibited directly through the action of specific toxins (as shown in the studies described above with $P$. aeruginosa in C. elegans and L. pneumophila in mammalian cells, and as previously observed by others ${ }^{22,25}$ ), and situations in which it is inhibited as a result of the induction of stress pathways that converge towards modulation rather than inhibition of translation. This point is also of particular importance because the $P$. aeruginosa toxin and L. pneumophila effectors that directly inhibit translation target the elongation step, whereas other bacterial pathogens that affect metabolic stress pathways have an impact on translation initiation (FIG. 1). Indeed, Chakrabarti et al. ${ }^{8}$ and Tattoli et al. ${ }^{14}$ used bacterial pathogens that do not encode toxins which directly target the translation machinery (P. entomophila in D. melanogaster, and S. flexneri and S. Typhimurium in human epithelial cell lines, respectively). Interestingly, both studies identified an effect of the pathogens on two key signalling axes known to affect host translation: the GCN2-eIF2 $\alpha$ and TOR pathways. Although it must be noted that Tattoli et al. ${ }^{14}$ did not investigate whether the induction of these pathways affects host translation, these results collectively imply that common host metabolic stress pathways are triggered in response to bacterial pathogens and in particular in response to host membrane damage triggered by either pore-forming toxins ${ }^{8,22}$ or bacterial secretion systems ${ }^{24}$. It is likely that the induction of the GCN2-eIF2 $\alpha$ and TOR pathways represents an adaptation to the cellular stress induced by bacterial pathogens or some toxins and probably results in global metabolic reprogramming, as these pathways have an impact not only on translation, but also on cell growth and proliferation, as well as on autophagy. Tattoli et al. showed that induction of the human GCN2-eIF2 $\alpha$ and mTOR pathways leads to ATF3-associated transcriptional reprogramming as well as autophagy against intracellular bacteria, thus suggesting that these responses contribute to host defence ${ }^{14}$. Chakrabarti et al. also demonstrated that, in D. melanogaster, Gcn2 and Tor contribute to host defence against the pathogenic but non-lethal bacterium Erwinia carotovora ${ }^{8}$. However, in the case of P. entomophila, the translation shut-off resulting from both metabolic stress and the build-up of reactive oxygen species contributed to the pathogenicity of the bacterium, suggesting that excessive induction of these host metabolic stress pathways can be detrimental to host defences. 
Two studies also revealed that inhibition of translation shapes the transcriptional profile of the innate immune response $e^{8,10}$. This coupling could be due to the fact that most innate immune pathways are tightly controlled by short-lived negative regulators, and these short-lived regulators are more sensitive to inhibition of translation than the immune effectors themselves. As a result, translation inhibition could actually result in increased innate immune transcriptional activity, which might maintain an effective immune response. Future studies should assess the global relevance of this idea as well as determine how the range of translation inhibition affects innate immunity.

Bacterial effectors and, notably, toxins affect the normal function of target cells, resulting in cellular adaptation and countermeasures that ultimately lead to either neutralization of the effect of the bacterial molecule or cell death. In plant ETI and in DAMP-associated immune activation, specific sets of sensors and receptors evolved to respond to cellular perturbations. In the case of plant ETI, these receptors detect either the bacterial effector itself or the effect of this molecule on basal plant defences, and as a result these receptors confer resistance to a unique bacterial pathogen (or a select set of bacterial pathogens). This explains why hundreds of resistance genes can be found in the genome of a given plant. In the case of DAMP-associated immune activation, a limited set of specific sensors evolved to detect broader cellular perturbations correlating with general cellular dysfunction, as exemplified by NLRP3 (NOD-, LRR- and pyrin domain-containing 3 ) in mammals, which detects potassium efflux or lysosomal membrane damage. In the absence of clear evidence that bacterium-induced translation inhibition operates through specific receptors, it is uncertain whether this translation inhibition represents a DAMP or whether it drives ETI. The interpretation that we favour is that translation inhibition is part of the stress response induced by pathogenic bacteria and should be considered a metabolic adaptation to maintain basic host cell functions despite the damaging effects of bacterial effectors or toxins. We predict that in the near future there will be more examples of how PRR-dependent pathways and cellular stress responses are integrated to shape host immunity and, more generally, the host response to bacterial pathogens.

In summary, the identification of a link between translation inhibition and host responses to bacterial pathogens highlights the key role of host metabolic stress pathways in innate immune defence. However, further investigation is required to determine whether translation inhibition represents a true direct sensor of bacterial pathogens or rather is a marker of a more general host metabolic stress response to the infection. Nevertheless, an important goal for future studies will be to better understand how cells infected with bacteria can maintain a plasticity of gene expression to allow immune defences to be mounted in the face of a global metabolic stress that dampens the activity of the global mRNA translation machinery.

Bruno Lemaitre is at the Global Health Institute, School of Life Science, École Polytechnique Fédérale de Lausanne (EPFL), 1015 Lausanne, Switzerland.

Stephen E. Girardin is at the Department of Laboratory Medicine and Pathobiology, University of Toronto, Toronto M5S 1A8, Canada.

e-mails: bruno.lemaitre@epfl.ch; stephen.girardin@utoronto.ca

doi:10.1038/nrmicro3029 Published online 13 May 2013

1. Kumar, H., Kawai, T. \& Akira, S. Pathogen recognition in the innate immune response. Biochem. J. 420, 1-16 (2009).

2. Fritz, J. H., Ferrero, R. L., Philpott, D. J. \& Girardin, S. E. Nod-like proteins in immunity, inflammation and disease. Nature Immunol. 7 , 1250-1257 (2006)

3. Tanji, T. \& Ip, Y. T. Regulators of the Toll and Imd pathways in the Drosophila innate immune response. Trends Immunol. 26, 193-198 (2005).

4. Takeuchi, O. \& Akira, S. Pattern recognition receptors and inflammation. Cell 140, 805-820 (2010).

5. Pedra, J. H., Cassel, S. L. \& Sutterwala, F. S. Sensing pathogens and danger signals by the inflammasome. Curr. Opin. Immunol. 21, 10-16 (2009).

6. Benko, S., Philpott, D. J. \& Girardin, S. E. The microbial and danger signals that activate Nod-like receptors. Cytokine 43, 368-373 (2008).

7. Bonardi, V. ¿ Dangl, J. L. How complex are intracellular immune receptor signaling complexes? Front. Plant Sci. 3, 237 (2012).

8. Chakrabarti, S., Liehl, P., Buchon, N. \& Lemaitre, B. Infection-induced host translational blockage inhibits immune responses and epithelial renewal in the Drosophila gut. Cell Host Microbe 12, 60-70 (2012).

9. Dunbar, T. L., Yan, Z., Balla, K. M., Smelkinson, M. G. \& Troemel, E. R. C. elegans detects pathogen-induced translational inhibition to activate immune signaling. Cell Host Microbe 11, 375-386 (2012).

10. Fontana, M. F. et al. Secreted bacterial effectors that inhibit host protein synthesis are critical for induction of the innate immune response to virulent Legionella pneumophila. PLoS Pathog. 7, e1001289 (2011).
11. Fontana, M. F., Shin, S. \& Vance, R. E. Activation of host mitogen-activated protein kinases by secreted Legionella pneumophila effectors that inhibit host protein translation. Infect. Immun. 80, 3570-3575 (2012).

12. McEwan, D. L., Kirienko, N. V. \& Ausubel, F. M. Host translational inhibition by Pseudomonas aeruginosa exotoxin A triggers an immune response in Caenorhabditis elegans. Cell Host Microbe 11, 364-374 (2012).

13. Melo, J. A. \& Ruvkun, G. Inactivation of conserved C. elegans genes engages pathogen- and xenobiotic-associated defenses. Cell 149 452-466 (2012)

14. Tattoli, I. et al. Amino acid starvation induced by invasive bacterial pathogens triggers an innate host defense program. Cell Host Microbe 11, 563-575 (2012).

15. Mohr, I. \& Sonenberg, N. Host translation at the nexus of infection and immunity. Cell Host Microbe 12, 470-483 (2012).

16. Luo, Z.-Q. Legionella secreted effectors and innate immune responses. Cell. Microbiol. 14, 19-27 (2012).

17. Hubber, A. \& Roy, C. R. Modulation of host cell function by Legionella pneumophila type IV effectors. Annu. Rev. Cell Dev. Biol. 26, 261-283 (2010).

18. Estes, K. A., Dunbar, T. L., Powell, J. R., Ausubel, F. M. \& Troemel, E. R. bZIP transcription factor zip-2 mediates an early response to Pseudomonas aeruginosa infection in Caenorhabditis elegans. Proc. Natl Acad. Sci. USA 107, 2153-2158 (2010).

19. Vattem, K. M. \& Wek, R. C. Reinitiation involving upstream ORFs regulates ATF4 mRNA translation in mammalian cells. Proc. Natl Acad. Sci. USA 101, 11269-11274 (2004).

20. Buchon, N., Broderick, N. A., Poidevin, M. Pradervand, S. \& Lemaitre, B. Drosophila intestinal response to bacterial infection: activation of host defense and stem cell proliferation. Cell Host Microbe 5, 200-211 (2009).

21. Buchon, N., Broderick, N. A., Kuraishi, T. \& Lemaitre, B. Drosophila EGFR pathway coordinates stem cell proliferation and gut remodeling following infection. BMC Biol. 8, 152 (2010).

22. Kloft, N. et al. Pro-autophagic signal induction by bacterial pore-forming toxins. Med. Microbiol. Immunol. 199, 299-309 (2010).

23. Kloft, N. et al. A subunit of eukaryotic translation initiation factor $2 \alpha$-phosphatase (CreP/PPP1R15B) regulates membrane traffic. J. Biol. Chem. 287 35299-35317 (2012).

24. Tattoli, I., Philpott, D. J. \& Girardin, S. E. The bacterial and cellular determinants controlling the recruitment of mTOR to the Salmonella-containing vacuole. Biol. Open 1, 1215-1225 (2012)

25. Gonzalez, M. R. et al. Pore-forming toxins induce multiple cellular responses promoting survival. Cell. Microbiol. 13, 1026-1043 (2011).

Acknowledgements

The authors thank E. R. Troemel and D. J. Philpott for critical reading of the manuscript. Research in the B.L. laboratory is supported by grants from the European Research Council and by the Swiss National Science Foundation (grant 3100A0-12079/1). Research in the S.E.G. laboratory is supported by grants from the Canadian Institutes of Health Research, the Burroughs Wellcome Fund and the Crohn's and Colitis Foundation of Canada.

Competing interests statement

The authors declare no competing financial interests.

\section{FURTHER INFORMATION}

Bruno Lemaitre's homepage: http://lemaitrelab.epfl.ch/ Stephen E. Girardin's homepage: http://www.Imp.utoronto. ca/research/faculty-research-database/girardin-stephen ALL LINKS ARE ACTIVE IN THE ONLINE PDF 


\title{
ONLINE ONLY
}

\section{TOC blurb}

\section{Translation inhibition and metabolic stress pathways in the host response to bacterial pathogens}

\author{
Bruno Lemaitre and Stephen E. Girardin \\ Recent studies have revealed a role for host \\ translation inhibition in the innate immune \\ surveillance and detection of bacterial pathogens. \\ Lemaitre and Girardin review these findings and \\ discuss whether translation inhibition is a direct \\ innate immune signal or rather part of a more general \\ metabolic stress response to infection.
}

\begin{abstract}
Author biographies
Bruno Lemaitre has been a professor at the École Polytechnique Fédérale de Lausanne, Switzerland, since 2007. He obtained his Ph.D. in 1992 at the Université Pierre et Marie Curie (Paris VI), France, and received postdoctoral training in the Centre National de la Recherche Scientifique (CNRS), Strasbourg, France. In 1998, he started his own laboratory at the Centre Génétique Moléculaire, a research institute of the CNRS at Gif-sur-Yvette, France. His laboratory uses the fruitfly as a model genetic system and carries out cutting-edge research in the field of innate immunity and hostpathogen interactions.
\end{abstract}

Stephen E. Girardin undertook his undergraduate studies at the École Normale Supérieure d'Ulm, Paris, France, and the Université Pierre et Marie Curie (Paris VI), and then studied JNK signal transduction pathways in the laboratory of Moshe Yaniv at the Pasteur Institute, Paris, obtaining his Ph.D. in 2001. He next joined the laboratory of Philippe Sansonetti, also at the Pasteur Institute, to study the host responses to intracellular bacterial pathogens. His worked contributed to the characterization of the role of NOD-like receptors in innate immunity. He obtained a faculty position at the Pasteur Institute in 2005 and was awarded an Institut National de la Santé et de la Recherche Médicale (INSERM) AVENIR junior group leader position the same year. He joined the Department of Laboratory Medicine and Pathobiology at the University of Toronto, Canada, in 2006 and is now Associate Professor. His laboratory uses cellular and in vivo models to study several aspects of the host response to intracellular bacterial pathogens. He is a recipient of a Burroughs Wellcome Fund award and holds a Canada Research Chair in innate immunity and microbial pathogenesis.

\section{Subject categories}

Bacteria

Innate immunity

Immune response 